\title{
An Eye Blink and Head Movement Detection for Computer Vision Syndrome
}

\author{
Vinaya Kulkarni \\ Department of Computer \\ Engineering, \\ Bharati Vidyapeeth's College of \\ Engineering for Women, \\ Dhankawadi, Pune, India
}

\author{
Chetana Thombre \\ Department of Computer \\ Engineering, \\ Bharati Vidyapeeth's College of \\ Engineering for Women, \\ Dhankawadi, Pune, India
}

\author{
Nehanaaz Shaikh \\ Department of Computer \\ Engineering, \\ Bharati Vidyapeeth's College of \\ Engineering for Women, \\ Dhankawadi, Pune, India
}

\author{
Tejashri Tarade \\ Department of Computer Engineering, \\ Bharati Vidyapeeth's College of Engineering for \\ Women, \\ Dhankawadi, Pune, India
}

\author{
Tejaswini Patne \\ Department of Computer Engineering, \\ Bharati Vidyapeeth's College of Engineering for \\ Women, \\ Dhankawadi, Pune, India
}

\begin{abstract}
The usage of computers in our day-to-day activities has increased enormously leading to both positive and negative effects in our lives. The negative effects are related to health problems such as Computer Vision Syndrome (CVS) etc. Prolonged use of computers would lead to a significant reduction of spontaneous eye blink rate due to the high visual demand of the screen and concentration on the work. The proposed system develops a prototype using blink as a solution to prevent CVS. The first part of the work captures video frames using web camera mounted on the computer or laptop. These frames are processed dynamically by cropping only the eyes, which determines the eye-status based on the threshold value and the proposed idea. Various experiments are done and their algorithms are compared and concluded that the proposed algorithm yields $99.95 \%$ accuracy.

Keywords: OpenCV, Computer Vision Syndrome, Haar Cascade.
\end{abstract}

\section{INTRODUCTION \\ Computer Vision Syndrome}

Computer became basic need for human. Almost all the task are completed using computer. These days, many of us have jobs that require us to stare at computer screens for hours at a time. That can put a real strain on your eyes. Medical study indicates an average adult blinks once in 4 seconds. When eye focuses on an object or pays more attention in activities such as reading, watching a video in a digitized environment the rate of blinking decreases to about 3 to 4 times per minute [2]. This transformation leads to a greater stress to the visual system leading to CVS .Also research shows that $40 \%$ to $80 \%$ have these symptoms. Eye problems caused by computer use fall under the heading computer vision syndrome (CVS). Prolong use of computer leads to, eye irritation, abnormal eye blink rate, head ache, blur eyes, neck and shoulder pain, itchy eyes, watery eyes.

The impact of the disease leads to poor visual functions, increased stress levels, reduced effective work hours, frequent absence from work, possible increase in errors, less time available for personal care and this in turn has reduced productivity. Thus confronting a solution has become the primary need. A lightweight product is expected with less execution time to adapt to the dynamic environment.

Amongst all these symptoms for computer vision syndrome, we are basically trying to focus on abnormal eye blink rate which will detect that the user has CVS and also focusing on the factor of abnormal head movement which shows that the user has neck pain, and also the third factor which is close eye duration which shows that the user is falling asleep due to strain in the eyes or constant use of computer. The proposed system will help to detect and prevent the symptoms of computer vision syndrome. The web camera captures the video and the frames are processed for detecting stress of eyes and head. OpenCv will first localize the head then will localize the eyes. OpenCv is also used for segmentation of head movement. Our system will calculate the eye blink rate, if it is greater or lesser than threshold value then Computer Vision Syndrome will be detected and for head movement detection if it is greater than threshold value then Computer Vision Syndrome will be detected. Then the system will generate the output as text to speech like stay alert or take a break.

\section{LITERATURE SURVEY}

2.1 Take-A-Break Notification by Nellmendee Julius, in 2014 is a software which runs on Windows operating system designed for office workers who have the highest tendency on prolonged computer screens use, in order to reduce Computer Vision Syndrome (CVS). The purpose of this study is to prevent computer users from looking in front of a computer screen for a long period of time. Rapid Application Development (RAD) methodology has been used for the project development phase.

2.2 Electrooculography Based Blink Detection Using Computer vision Syndrome by Monalisa Pall, Anwesha Banerjee in 2014 which proposes an artificial system capable of preventing Computer Vision Syndrome from the analysis of eye movements. Ocular data is recorded using an Electrooculogram signal acquisition system developed in the laboratory. Wavelet detail coefficients obtained using Haar 
and Daubechies order 4 mother wavelets are used as signal features. From the recorded data, blinks are classified from any other type of eye movements using Support Vector Machine ( SVM) classifier with different kernel functions. It obtain a maximum average accuracy of $95.83 \%$ over all classes and participants using second order polynomial kernel SVM classifier. Then the trained classifier has been used in real time to detect blinks. The system is designed to count the number of blinks in a particular interval of time thereby reminding people working on a computer for long periods to rest and blink frequently in case of insufficient number of blinks.

\subsection{Close Faced-Distance Warning System for computer} Vision Syndrome in 2015, Takeshi Toda, MasetoNakai, Xinxin Liu in which it proposes a close face-distance warning system in order to keep personal computer (PC) user away from to the PC display and to prevent straightened neck during prolong PC usage. The system estimates the distance between the display and the user face (called as face distance) from the user face area that is real-timely measured from captured image with built-in webcam. Haar-like cascade classifier is used for the facedetection and tracking from captured image every frame. The face distance is then estimated from the number of pixels of the detected skin color area. The system then shows a pop-upwarning message on the display when the face comes close to the display than a limit, in order to promote keeping face away from the display.

2.4 Web-based Content Management System Payal Wasnik, Amutha Jeyakumar have done a paper in 2016. In this, it remotely monitoring a patient's health condition is now easily possible with the use of sensors, actuators and mobile communication devices, combined together called as Internet of Things for Medical Devices. The ArduinoYun is a Microcontroller board having built-in Ethernet and Wi-Fi support and can be connected to a vast array of web-based Resource and services with the use of Temboo, a cloud-based platform with processes for APIs, databases, and more. The parameters considered under Ergonomic workspace like distance between user and computer, angle with which the computer screen should be positioned can be analysed using Ultrasonic And Accelerometer sensors; while pulse sensor to analyse heart rate under work pressure

\section{PROPOSED SYSTEM}

In this system, we have basically three modules,

\subsection{Head Movement Detection :}

Web Camera will capture the video and then it will convert these video into number of frames. Harcascade Algorithm is used for detection of head and eyes in this system. Using this frames OpenCV will localize the head and will localize the eyes. OpenCV is nothing but open source computer vision library, which is used for localization of moving object. This will give more clear images than colour models. It also used for segmentation of images. After localizing head, the system will calculate the head movement. We fixed the threshold value as $>5$ times in half an hour, that means if the end user will move his head more than 5 times in a such a way that it will cross the fixed range. This range is fixed by segmentation by using OpenCV. We consider left hand side and the right hand side range for segmentation. We will localize head and consider square area as per the user head size; this area will be range for detection of the head movement. If the head movement is not greater than threshold value, system will shift to next image. If the head movement is greater than threshold value then system will generate the output as take a break and be alert.

\subsection{Eye Movement Detection :}

The second part is detection of eyes. Using OpenCV we localize head and then will localize eyes. Until and unless the head is not localized the eye is not detected. In this modules there are two subparts where we consider the time of eye closed duration and blink rates. Normally the human eye blinks every 4 seconds. If the user will blink his eyes more or less than threshold value as 2-3 blinking per seconds then it will detect as computer vision syndrome. Because of low blinking rate user will face the eyes problems such as dry eyes, red eyes, and itchiness in eyes which are symptoms of computer vision syndrome. So if blinking rate is less or more than threshold value system will alert generate as take a break.

\subsection{Eye Close Duration:}

Sometimes, the blinking rate is very much low as user may get sleep while doing work on computer. So this will detect by the subpart as finding closed eye duration. If the end user will close his eyes more than 10 minutes, system will generate alert as be awake, stay alert. This will basically help the user to stay awake and do his/her work. So after getting alert from the system the end user will take break for some time or will more focus on work.

\section{SYSTEM ARCHITECTURE}

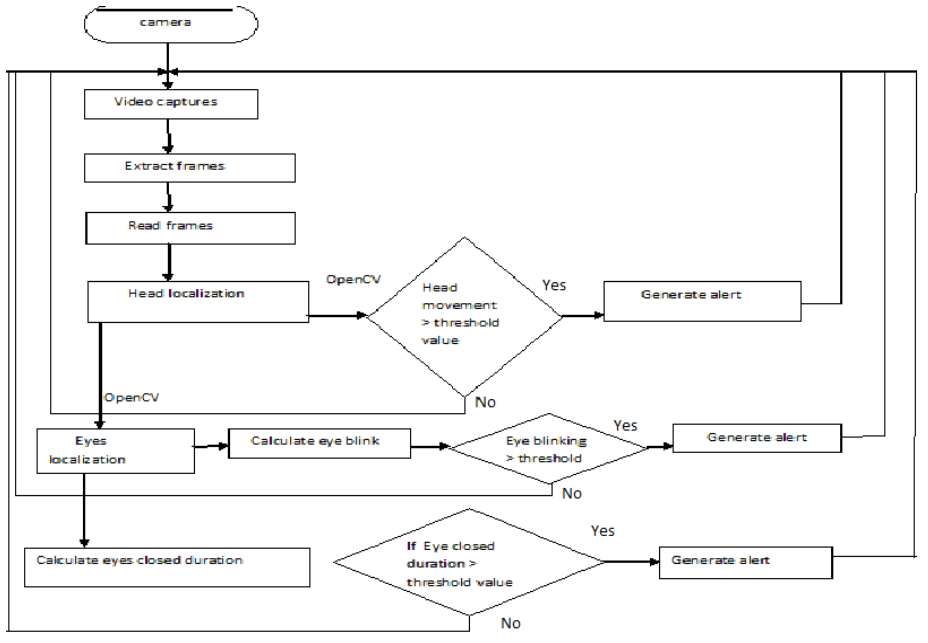




\section{Fig 1.System Architecture}

In System architecture, basically it shows :

4.1 Camera: It captures the video of an user who is sitting in front of the computer. Video gets captured and gets stored in the memory.

4.2 Frames Extraction: Once the video gets captured, the frames gets extracted from the video, and then are further processed.

4.3 Head Localization: Head is localized once the frame gets read , OpenCV localizes the head, if the head movement is abnormal, i.e. if it is more than the threshold value then the Computer Vision Syndrome is detected. It will then generate the alert, if it not detected then it will start to read the new frame.

4.3 Eye Localization: Head is localized once the frame gets read, OpenCV localizes the head and the eyes, if the eye movement is abnormal, ie if it is more than or less than the threshold value then the Computer Vision Syndrome is detected. It will then generate the alert, if it not detected then it will start to read the new frame again.

4.4 Closed Eye duration: Once the eye localization is done, if the users eye remains close more than the threshold value the, user gets an alert to stay awake or asked to take a break .

4.5 Generate Alert: Alert is generated if the computer Vision syndrome is detected.

\section{Haar Cascade Algorithm}

Object Detection using Haar feature-based cascade classifiers is an effective object detection method proposed by Paul Viola and Michael Jones in their paper, "Rapid Object Detection using a Boosted Cascade of Simple Features" in 2001. It is a machine learning based approach where a cascade function is trained from a lot of positive and negative images. It is then used to detect objects in other images.Here we will work with face detection. Initially, the algorithm needs a lot of positive images (images of faces) and negative images (images without faces) to train the classifier. Then we need to extract features from it. For this, haar features shown in below image are used. They are just like our convolutional kernel. Each feature is a single value obtained by subtracting sum of pixels under white rectangle from sum of pixels under black rectangle.
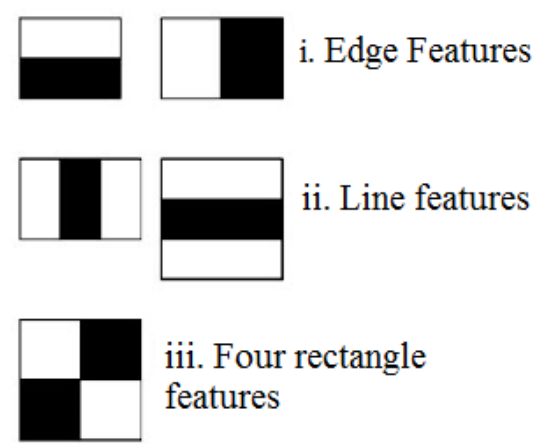

iii. Four rectangle

features

Fig 2. Haarcascade classifier

Now all possible sizes and locations of each kernel is used to calculate plenty of features. For each feature calculation, we need to find sum of pixels under white and black rectangles. To solve this, they introduced the integral images. It simplifies calculation of sum of pixels, how large may be the number of pixels, to an operation involving just four pixels. It makes things super-fast. But among all these features we calculated, most of them are irrelevant. For example, consider the image below. Top row shows two good features. The first feature selected seems to focus on the property that the region of the eyes is often darker than the region of the nose and cheeks. The second feature selected relies on the property that the eyes are darker than the bridge of the nose. But the same windows applying on cheeks or any other place is irrelevant. So how do we select the best features out of $160000+$ features? It is achieved by Adaboost.

\section{Haar-cascade Detection in OpenCV}

OpenCV comes with a trainer as well as detector. If you want to train your own classifier for any object like car, planes etc. you can use OpenCV to create one. Here we will deal with detection. OpenCV already contains many pre-trained classifiers for face, eyes, smile etc. Those XML files are stored in opencv/data/haarcascades/ folder. Let's create face and eye detector with OpenCV.

\section{Cascade Classifiers}

The object recognition process (in our case, faces) is usually efficient if it is based on the features take-over which include additional information about the object class to be taken-over. In this tutorial we are going to use the Haar-like features and the Local Binary Patterns (LBP) in order to encode the contrasts highlighted by the human face and its spatial relations with the other objects present in the picture. Usually these features are extracted using a Cascade Classifier which has to be trained in order to recognize with precision different objects: the faces' classification is going to be much different from the car's classification.

\section{ACKNOWLEDGMENTS}

We would like to take this opportunity to express sincere thanks to the department and the University for this Course where we have such an opportunity to express our ideas and put our learning all the way into practice.

\section{REFERENCES}

[1] Sofia Jennifer J, Sree Sharmila T "Edge based Eye Blink detection For Computer Vision Syndrome" April 2017

[2] Takeshi Toda, Masako Nakai, Xinxin Liu, "A Close Face-Distance Warning System For Straightened Neck Prevention" November 2015

[3] Monalisa Pal, Anweesha Banerjee, "Electrooculography Based blink detection to computer Vision Syndrome" November 2014

[4] A. Banerjee, S. Chakraborty, P. Das, S. Datta, A. Konar, D.N .Tibarewala and R. Janarthanan, "Single channel electrooculogram(EOG) based interface for mobility aid" , Fourth International Conference on Intelligent Human Computer Interaction, 2012.

[5] Seongwon Han, Sungwon Yang, Jihyoung Kim and Mario Gerla , "EyeGuardian: A Framework of Eye Tracking and Blink Detection forMobile Device Users", HotMobile12 February 2829, 2012, San Diego,CA, USA. 